\title{
Postprint Version
}

G. McHale, N.J. Shirtcliffe, C.R. Evans and M.I. Newton, Terminal velocity and drag reduction measurements on superhydrophobic spheres, Appl. Phys. Letts. 94 art. 064104. (2009); DOI: 10.1063/1.3081420

The following article appeared in Applied Physics Letters and may be found at http://link.aip.org/link/?APPLAB/94/064104/1. This article may be downloaded for personal use only. Any other use requires prior permission of the author and the American Institute of Physics. Copyright (02009 American Institute of Physics.

\section{Terminal velocity and drag reduction measurements on superhydrophobic spheres}

\author{
G. McHale*, N. J. Shirtcliffe, C. R. Evans and M. I. Newton \\ School of Science and Technology, \\ Nottingham Trent University,
}

Clifton Lane, Nottingham NG11 8NS, UK

\begin{abstract}
Super water-repellent surfaces occur naturally on plants and aquatic insects and are created in the laboratory by combining micro or nano-scale surface topographic features with hydrophobic surface chemistry. When such types of water-repellent surfaces are submerged they can retain a film of air (a plastron). In this work, we report measurements of the terminal velocity of solid acrylic spheres with various surface treatments settling under the action of gravity in water. We observed increases in terminal velocity corresponding to drag reduction of between $5 \%$ and $15 \%$ for superhydrophobic surfaces that carry plastrons.
\end{abstract}

\footnotetext{
* To whom correspondence should be addressed. E-mail: glen.mchale@ntu.ac.uk
} 
A droplet of water placed on a smooth hydrophobic Teflon ${ }^{\circledR}$ surface adopts a hemispherical shape. For the droplet to completely ball up and run-off the surface requires the water repellency provided by the surface chemistry to be amplified by the effect of surface topography. By creating a structure that appears to a water droplet as a bed-of-nails (or fakir's carpet) so that the surface becomes superhydrophobic, droplets can be made to roll freely. ${ }^{1-3}$ Recently, many man-made materials have been designed to mimic these effects and so create simple and functional, selfcleaning, water-repellent surfaces. ${ }^{4} \mathrm{~A}$ wide range of researchers have also speculated and investigated whether such superhydrophobic surfaces may also provide a mechanism to create a slip boundary condition and so reduce drag on fluids by solid walls. ${ }^{5}$ It has been speculated that drag may be reduced when water flows across superhydrophobic surfaces and results from plates and microfluidic channels suggest some drag reduction might exist. However, these systems have mostly considered flow through microchannels or use of plates within, e.g. cone-and-plate rheometers, and some results have been the subject of controversy. In this report, we explore the possibility of drag reduction using a simple terminal velocity experiment using small solid spheres. As a sphere settles in water it will reach a terminal velocity, $U_{T}$, determined by the drag of the surrounding water and this is not normally dependent on the surface chemistry of the object. In the present study, we show how coating surfaces with hydrophobic sand can reduce drag in a classic terminal velocity experiment. This reduction can be explained by considering the effect of a plastron film of air retained at the surface of some super water-repellent materials. The understanding gained introduces a new factor into the design of drag reducing surfaces and has wide applicability.

In our experiments, we used a column of water contained within a large transparent acrylic cylinder $(0.65 \mathrm{~m}$ diameter and $2.2 \mathrm{~m}$ height). Falling objects were created from commercially available acrylic spheres of nominal diameters (in imperial units) of 1", 11/2" and 2" (measured values of $2.533 \mathrm{~cm}, 3.816 \mathrm{~cm}$ and $5.071 \mathrm{~cm}$ with an accuracy of $\pm 50 \mu \mathrm{m})$ and typical masses of $10.1 \mathrm{~g}, 34.5 \mathrm{~g}$ and $83.6 \mathrm{~g}$. A camcorder operating at 30 frames per second was located $1 \mathrm{~m}$ below the top of the surface of water and used to film the spheres as they fell through the column of water and the terminal velocity calculated from the video analysis (Fig. $1)$; all terminal velocities reported are averages from at least eight drop experiments. Since the spheres fell with a slight side-to-side oscillatory trajectory the velocities provided in this report are the vertical components of terminal velocity; although not reported here we also conducted experiment using weighted spheres with cone shaped ends and these fell with a vertical trajectory. To verify the accuracy of the measurement approach, a separate set of experiments was conducted using a laser beam triggered timing gate system positioned so that the speed of falling objects could be calculated over two consecutive depths. From these
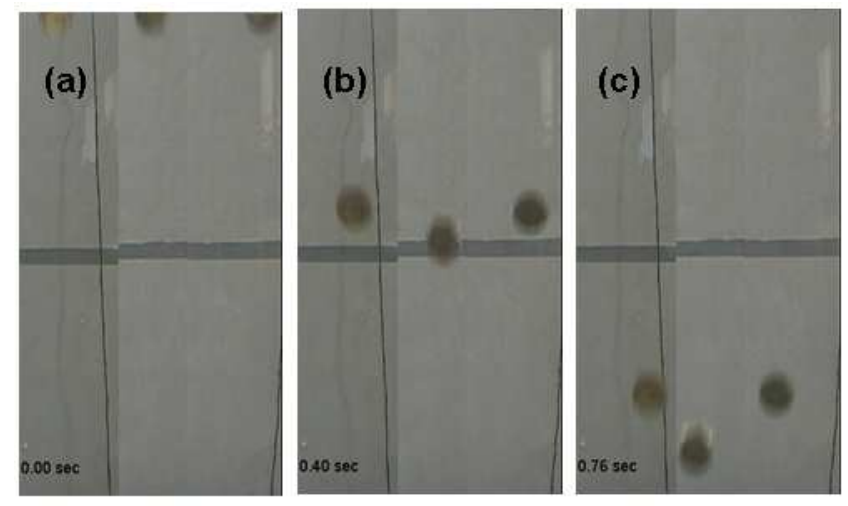

FIG. 1. A sequence of three images (a-c) showing a comparison of settling in water of a $1 \frac{1 / 2}{2}$ inch diameter acrylic sphere with three different surface coatings. Each image consists of a composite of three frames from three different experiments; the sequence of frame selected is the one corresponding to the median terminal velocity for that surface coating from a set of ten drop tank experiments. The object on the left in each image is an acrylic sphere with a thin layer coating of sieved sharp sand with grain sizes below $50 \mu \mathrm{m}$. The object in the centre in each image is the same acrylic sphere with its thin layer coating of sieved sharp sand, but with an additional coating of the HIREC superhydrophobic paint. The object on the right in each image is the same as in the centre, but with an ethanol pre-treatment to suppress the formation of a plastron. 
arrangements we confirmed that terminal velocity had been achieved. Typical terminal velocities were $\sim 0.42-0.56 \mathrm{~m} \mathrm{~s}^{-1}$ giving Reynolds numbers of $R e \sim 1 \times 10^{4}-3 \times 10^{4}$; these Reynold's numbers are well above those for which Stokes law would be valid.

To determine the effect of extreme water-repellence, the surfaces of the spheres were treated with a range of hydrophobic and superhydrophobic coatings and contact angles and contact angle hysteresis measured using a Krüss contact angle meter. To create a base surface topography for superhydrophobicity, we used a coating of sharp sand, sieved to give three grain sizes, $300 \mu \mathrm{m}$ to $350 \mu \mathrm{m}, 100 \mu \mathrm{m}$ to $150 \mu \mathrm{m}$ and below $50 \mu \mathrm{m}$. These layers were subsequently treated with either HIREC-1450 (super)hydrophobic spray paint or a water-proofing agent designed for GoreTex ${ }^{\circledR}$ clothing (Granger's Extreme Wash-in) to provide a hydrophobic surface chemistry; we also used commercially available superhydrophobic sand (Magic Sand ${ }^{\mathrm{TM}}$ ) coated directly onto the acrylic spheres. For comparison, experiments using the HIREC-1450 and the Granger's Extreme Wash-in coated directly onto the acrylic spheres were performed. The blank (acrylic) surface gave a contact angle of $(65 \pm 5)^{\circ}$ and the sharp sand gave $0^{\circ}$ on all grain sizes. The HIREC-1450 (super)hydrophobic spray paint gave a high contact angle of $(150 \pm 10)^{\circ}$ with low hysteresis even when sprayed directly onto a smooth surface. All hydrophobic sand-coated surfaces had contact angles in excess of $150^{\circ}$ and showed low contact angle hysteresis.

All superhydrophobic-coated spheres were observed to have a silvery sheen when submersed in water, indicative of a film of air (a plastron) retained at their surfaces. ${ }^{6,7}$ A plastron structure, used by some aquatic insects to extract oxygen directly from water without the need for gills, is a superhydrophobic surface that also conveys the additional property of fixing to the body a thin film of air. ${ }^{8-10}$ To compare the effect on terminal velocity of spheres with and without this surface retained film of air we developed a wetting out procedure to prevent the formation of a plastron. Immersing a superhydrophobic sphere into ethanol causes the liquid to come fully into contact with the grains of sand coating the surface of the sphere. Once a sphere had been fully wetted due to the ethanol, it was transferred into a container of water thus ensuring the surface remained completely wetted, but with the ethanol having been replaced by water. Drop testing of these pre-wetted spheres in the column of water allowed terminal velocity measurements to be made with nonplastron bearing hydrophobic sand-coated spheres.

In our experiments we did not observe any significant differences in terminal velocity between the bare acrylic surfaces and the acrylic surfaces treated with either of the hydrophobic coatings; it was visually noticeable that an acrylic sphere with the HIREC-1450 coated directly onto it without a sand-coating did not possess a plastron. However, we did observe an increase in terminal velocity when plastron-bearing superhydrophobic spheres were drop tested. As an example, when a thin layer of sieved sharp sand with grain sizes below $50 \mu \mathrm{m}$ or between 100-150 $\mu \mathrm{m}$ was coated onto a sphere, only a small increase in terminal velocity was observed; this is consistent with a small overall increase in density for the sphere. However, when the same sphere was subsequently treated with the water-proofing agent designed for GoreTex ${ }^{\circledR}$ clothing (Granger's Extreme Wash-in) and the terminal velocity measured again, a significant increase was observed (Fig. 2a-c). The same results were observed for the Hirec-1450 treated sand-coated spheres. Drop testing of pre-wetted spheres in the column of water allowed terminal velocity measurements to be made with non-plastron bearing hydrophobic sand-coated spheres. In all cases, the measured terminal velocity for the non-plastron bearing spheres returned to values close to those observed for 
the sand-coated spheres prior to their hydrophobic treatment. This method provides a direct comparison of the terminal velocity of any individual superhydrophobic sphere with and without a plastron (Fig. 2a-c). To confirm the effectiveness of this method, we also coated our spheres with commercially available hydrophobic sand, Magic Sand ${ }^{\mathrm{TM}}$ and increases in terminal velocity were observed in water when the spheres carried a plastron (Fig. 2d).

To convert terminal velocities to a drag force, $F_{d}$, on a sphere consider $F_{d}=1 / 2 C_{d} \rho_{f} A U^{2}$ where $\rho_{f}$ is the density of the fluid, $A$ is the cross-sectional area of the sphere, $U$ is the speed of the fluid relative to the solid and $R e$ is the Reynolds number, which gives a measure of the ratio of inertial forces to viscous forces. ${ }^{11}$ In general, the coefficient of drag depends on the Reynold's number and the surface roughness. For our Reynold's number range $\left(\operatorname{Re} \sim 1 \times 10^{4}\right.$ to $\left.3 \times 10^{4}\right)$ the co-efficient of drag is inversely proportional to the square of the terminal velocity (i.e. $C_{d} \propto 1 / U_{T}{ }^{2}$ ). Moreover, a superhydrophobic sphere tested with and without the ethanol wetting out procedure has no change in surface roughness. Therefore, the effect of the plastron on drag can be estimated from $C_{d}{ }^{p}=C_{d}{ }^{e}$ $\left(U_{T}^{e} / U_{T}^{p}\right)^{2}$, where the superscript $p$ indicates a plastron bearing sphere and the superscript $e$ indicates the same sphere with the ethanol pre-treatment to prevent formation of the plastron. In our experiment using a settling sphere, the additional buoyancy due to the air within a plastron means that our estimate of the reduction in drag is an underestimate. From this we deduce that the plastron induced by superhydrophobic sand reduces the coefficient of drag by between $5 \%$ and $15 \%$. Fig. 3a shows both the accuracy for repeated experiments with any given sample (error bars) and reproducibility between samples (representing surface preparation and sphere uniformity). To estimate the thickness of plastrons induced on the spheres, we prepared glass microscope slides with the same hydrophobic sand coatings of various grain sizes. By first immersing these slides in a container filled with water and then with the container filled to the same level with ethanol, which completely wets the hydrophobic sand, and measuring masses we estimated the thickness of the resulting plastrons. The thickness of the plastron increased with grain size fraction from tens of microns to hundreds of microns (Fig. 3b); these estimates were consistent with values observed with a travelling microscope.
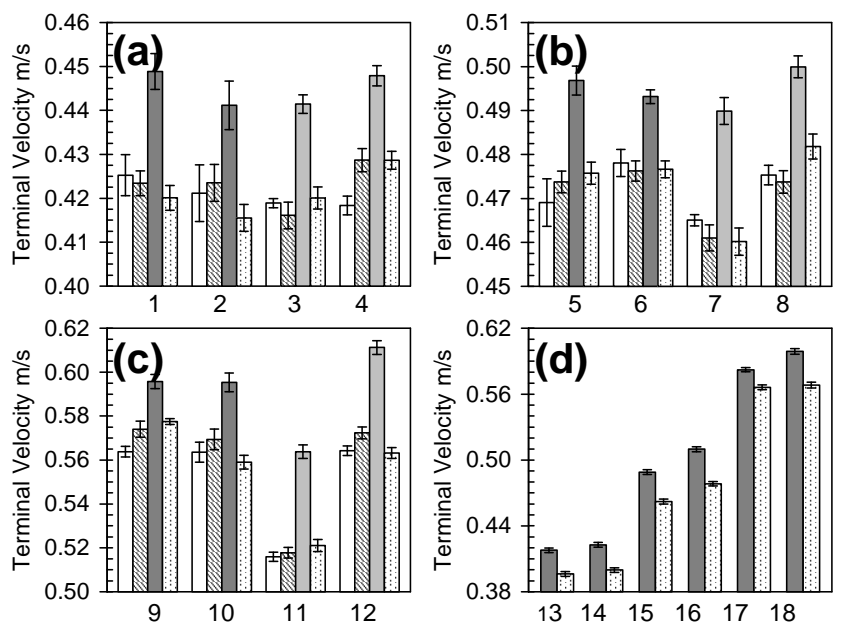

Fig. 2. Terminal velocities (vertical components) measured for spheres with and without hydrophobic sand surface finishes. (a) Four spheres with 1 inch diameter and various surface finishes. The first group of four bars shows one sphere with the following sequence: (i) blank surface (unfilled bar), (ii) surface coated with sieved sand of below $50 \mu \mathrm{m}$ grain size (diagonal pattern bar), (iii) Granger's Gore-Tex ${ }^{\circledR}$ waterproofing treated sand surface (shaded bar), and (iv) Granger's Gore-Tex ${ }^{\circledR}$ waterproofing treated sand surface with ethanol pre-treatment to prevent formation of a plastron (dotted bar); each bar represents the average terminal velocity from at least eight drop tank measurements. The second group of four bars is a replicate of the same sequence using a new sphere. The third group of four bars uses a superhydrophobic paint (HIREC-1450) to impart water repellency (lighter shaded bar) and the fourth group of four bars is a replicate of the same sequence using a new sphere. (b) and (c) are repeats of (a) using 1 1/2 inch and 2 inch diameter spheres, respectively. (d) shows the terminal velocity in water (shaded bar) and then in water, but with an ethanol pre-treatment to prevent the formation of a plastron (dotted bar), for two spheres of each diameter coated in commercially available superhydrophobic Magic Sand ${ }^{\mathrm{TM}}$. 
To understand why a reduction in drag might be associated with the formation of a plastron, first consider a low Reynold's number terminal velocity experiment with a solid sphere. If this solid sphere is replaced by a bubble of gas of the same diameter the Stokes' drag with a drag coefficient $C_{d}=24 / R e$ is replaced by Hadamard-Rybczynski drag with a reduced drag coefficient $C_{d}=16 / R e$, which is $33 \%$ lower. $^{11}$ This is because the replacement of the solid-water interface by an air-water interface allows the tangential stress from the external flow to induce an internal circulation within the bubble. A similar situation is known to occur in complex
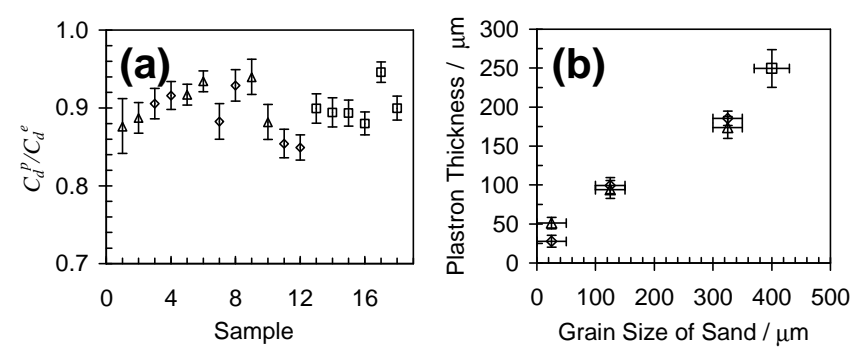

Fig. 3. (a) Estimates from the data in fig. 2 of the ratio of drag coefficients with and without plastron using the terminal velocity measured with the plastron bearing sphere in water and the same sphere using an ethanol pre-treatment to prevent the formation of a plastron. (b) Thickness of the plastron in water measured on sieved fractions of sand possessing i) a HIREC-1450 hydrophobic paint coating (diamonds), ii) a Granger's Gore-Tex ${ }^{\circledR}$ waterproofing (triangles), and iii) commercially available superhydrophobic Magic Sand ${ }^{\mathrm{TM}}$ (squares). compound multiphase droplets constructed with an outer fluid shell and an inner core fluid, socalled encapsulated droplets. Rushton and Davies derived an analytical solution for a spherical core fluid with a concentric spherical fluid shell, which predicted that both the core and the shell have internal circulations and so result in a correction to Stokes' drag. ${ }^{12}$ When the concentric fluid shell consists of air, the correction factor tends to the Hadamard-Rybzcynski bubble correction factor provided the thickness of the shell does not become vanishingly small, thus preventing circulation of air within the concentric spherical shell surrounding the solid core. At higher flow speeds, with intermediate Reynolds number, such as in our experiments, the wake behind a solid particle changes from an attached laminar wake to a separated wake, and ultimately to a turbulent wake. However, the internal circulation within a bubble possessing an uncontaminated interface to the liquid changes the wake separation of the external liquid. ${ }^{13}$ The consequent reduction in drag compared to a solid sphere has been reported in the literature for a fluid film encapsulated spherical fluid droplet using numerical simulations with $R e=200-1000 .{ }^{14}$ These considerations indicate that drag reduction might require not just a superhydrophobic coating, but also one which retains a sufficiently thick plastron. This would explain why the HIREC paint, which derives its superhydrophobicity from the inclusion of small Teflon particles of around $400 \mathrm{~nm}$ diameter, does not result in drag reduction when applied directly to an acrylic sphere, but does when applied on top of a layer of larger sand grains. These considerations introduce a new factor determining frictional drag, which is the combined effect of surface chemistry and surface topography.

In conclusion, our results have demonstrated that surface coatings can be designed using a suitable combination of hydrophobicity and small-scale surface structure to reduce frictional drag in liquids. The concept is simple, and powerful, requiring only a non-wetting surface that retains a film of air. This implies that whilst some aquatic insects have naturally evolved plastrons to breathe underwater, a similar structure can also be engineered to decrease drag. Plastron drag reduction concepts are promising for all situations in which more efficient transport of a solid through a liquid, or of a liquid across a solid surface, is required.

\section{Acknowledgement}

The authors' acknowledge the financial support of the UK EPSRC under grant EP/D500826/1. 


\section{References}

1. T. Onda, S. Shibuichi, N. Satoh and K. Tsujii, Langmuir 12, 2125 (1996).

2. W. Barthlott and C. Neinhuis, Planta 202, 1 (1997).

3. D. Quéré, A. Lafuma and J. Bico, Nanotechnology 14, 1109 (2003).

4. P. Roach, N. J. Shirtcliffe and M. I. Newton, Soft Matter 4, 224 (2008).

5. R. S. Voronov, D. V. Papavassiliou and L. L. Lee, Ind. Eng. Chem. Res. 47, 2455 (2008).

6. N. J. Shirtcliffe, G. McHale, M. I. Newton, C. C. Perry and F. B. Pyatt, Appl. Phys. Lett. 89, art. 104106 (2006).

7. M. R. Flynn, J. W. M. Bush, J. Fluid Mech. 608, 275 (2008).

8. W. H. Thorpe and D. J. Crisp, J. Exp. Biol. 24, 227 (1947).

9. D. J. Crisp and W. H. Thorpe, Faraday Soc. Discuss. 3, 210 (1948).

10. W. H. Thorpe, Biol. Rev. 25, 344 (1950).

11. L. D. Landau and E. M. Lifshitz, Fluid mechanics, London, Pergamon Press (1959).

12. E. Rushton and G. A. Davies, Int. J. Multiphase Flow 9, 337 (1983).

13. E. Loth, Int. J. Multiphase Flow 34, 523 (2008).

14. S. Kawano and H. Hashimoto, JSME Int. J. Ser. II 35, 151 (1992). 